Original Research Paper

\title{
Influence of Flow Direction on Vortex Flow Structure, Heat Transfer and Performance Improvement in Tube with $\mathrm{V}$ - Discreted Baffles
}

\author{
${ }^{1}$ Withada Jedsadaratanachai and ${ }^{2}$ Amnart Boonloi \\ ${ }^{I}$ Department of Mechanical Engineering, Faculty of Engineering, King Mongkut's Institute of Technology Ladkrabang, \\ Bangkok 10520, Thailand \\ ${ }^{2}$ Department of Mechanical Engineering Technology, College of Industrial Technology, King Mongkut's University of \\ Technology North Bangkok, Bangkok 10800, Thailand
}

Article history

Received: 13-11-2013

Revised: 20-11-2013

Accepted: 26-11-2014

Corresponding Author: Amnart Boonloi

Department of Mechanical

Engineering Technology,

College of Industrial

Technology, King Mongkut's

University of Technology

North Bangkok, Bangkok

10800, Thailand

Email: amnartb@kmutnb.ac.th

\begin{abstract}
Nowadays, the compact heat exchanger is used widely like a radiator, evaporator and condenser of air condition system, cooling device of electronic. Extensive studies have been done on the heat transfer characteristics and flow structure for the heat exchanger with longitudinal vortex generators by numerical method. Periodic laminar flow and heat transfer characteristics in a three-Dimensional (3D) isothermal circular tube wall with $45^{\circ}$ in-line Vdiscrete baffles were investigated numerically. The computations are based on the Finite Volume Method (FVM) and the SIMPLE algorithm has been implemented. The fluid flow and heat transfer characteristics are shown for Reynolds numbers based on the diameter of the tube, $R e=100$ to 1200 . To generate main longitudinal vortices flows through the tested section, V-discrete baffles with an attack angle of $45^{\circ}$ are mounted in tandem with in-line arrangement, pointing downstream (V-Downstream) and pointing upstream (VUpstream) inserted in the middle of the tested circular tube. Effects of Different Blockage Ratio (b/D, BR) with a single Pitch Ratio (P/D, PR) of 1 on heat transfer and pressure loss in the circular tube are studied. It is apparent that in each of the main vortex flows, longitudinal twisted vortex flows can induce impinging flows on a wall of the inter baffle cavity leading to a drastic increase in heat transfer rate over the circular tube. In addition, the rise in the V-baffle height results in the increase in the Nusselt number and friction factor values. The flow structures, common-flow-up and common-flow-down are appeared by using a baffle with V-Downstream and V-Upstream, respectively. The computational results show that the optimum thermal enhancement factor is around 2.75 at $\mathrm{BR}=0.10, \mathrm{Re}=1200$ for $\mathrm{V}$-Upstream discrete baffle.
\end{abstract}

Keywords: Periodic Flow, Laminar Flow, Heat Transfer, V-Discrete Baffle, V-Downstream, V-Upstream

\section{Introduction}

The required for more efficient and performance improvement of heat exchanger had been done by increase heat transfer rate with turbulators or vortex generators. Many types of turbulators such as rib, fin, groove, baffle, winglet, etc. were installed into a tube or channel of heat exchanger to increase higher level of turbulence vortex flow and increase degree of impinging jet flow over the walls of the channel. The impinging jet of flow in a heat exchange system can be augmented not only heat transfer rate but also increase in pressure loss.
The designs of turbulators and their parameters; shape, attach angle, height and space have been interested. Array of V-shaped baffle were used to insert into the channel or tube in heat exchanger to create impinging jet on the wall leads to the increase in heat transfer rate and efficiencies. The Higher of Blockage Ratio (b/H, BR) of V-baffle give the highest heat transfer rate but also provide very enlarge pressure loss. To reduce the pressure loss of the system, the investigation of Pitch spacing or Pitch Ratio (P/H, PR) was reported by (Promvonge et al., 2010; 2012). The results showed that the rise of PR leads to the decrease on both pressure loss and heat transfer. Han and Zhang (1992) 
experimentally investigated the effect of 45 and $60^{\circ}$ parallel ribs and V-shaped ribs in the square channel. They reported that all of the turbulators increase in heat transfer rate with not as much of the rise up flow resistance. Taslim et al. (1996) experimentally investigated oblique ribs and Vshaped ribs. They showed that the high heat transfer rate cause of the secondary flows that generated by oblique ribs and V-shaped ribs. The experimental examination of the heat transfer coefficient in rib-roughened channels with continuous ribs, interrupted ribs and V-shaped interrupted ribs were reported by (Giovanni, 2004). The experimental results indicated that the shape of turbulators have effect for flow structure and heat transfer behavior and also referred that the transverse interrupted ribs give the highest heat transfer. The experimental investigation of V-shaped and angled ribs in rotating rectangular channels by (Lee et al., 2005). They also confirmed that the V-shaped rib gave better heat transfer enhancement than the angled rib configurations. Fu et al. (2006) showed the related results. They reported that the V-shaped ribs and the discrete Vshaped ribs provide better heat transfer enhancement. Peng et al. (2011) studied on both experimentally and numerically for convection heat transfer in channels with different types of ribs; $90^{\circ}$ continuous rib, $90^{\circ}$ interrupted rib, $45^{\circ} \mathrm{V}$-shaped continuous rib, $45^{\circ} \mathrm{V}$-shaped interrupted rib, $60{ }^{\circ} \mathrm{V}$-shaped continuous rib and $60^{\circ} \mathrm{V}$-shaped interrupted rib. The results indicated that the $45^{\circ} \mathrm{V}$-shaped continuous ribs have the highest thermal performance. They also reported the comparison of continuous and interrupted ribs; the V-shaped interrupted ribs give lower heat transfer rate than that from the $\mathrm{V}$-shaped continuous ribs, while the $90^{\circ}$ ribs had the opposite result. Effects of Staggered discrete V-apex up (V-Upstream) and down (VDownstream) rib and transverse staggered discrete ribs on heat transfer were reported by (Muluwork, 2000). The results showed that the $\mathrm{V}$-down discrete ribs performed better in comparison with V-up and transverse discrete ribs. Momin et al. (2002) displayed that the $60^{\circ} \mathrm{V}$-ribs give higher heat transfer rate and pressure loss than inclined ribs. The heat transfer of rotating rectangular duct with compound scaled roughness and V-ribs at high rotation numbers was reported by Chang et al. (2009). Chang et al. (2008) also reported the heat transfer and pressure drop in a rectangular channel with the compound roughness of $\mathrm{V}$ shaped ribs and deepened scales. Karwa and Chitoshiya (2013) experimentally studied of thermo-hydraulic performance of a solar air heater with $60^{\circ} \mathrm{V}$-down discrete rib roughness on the air flow side of the absorber plate which, the enhancement in the thermal efficiency due to the roughness on the absorber plate is found to be $12.5-20 \%$ depending on the airflow rate; higher enhancement is at the lower flow rate. Singh et al. (2012) studied on thermohydraulic performance caused by flow-attack-angle in Vdown rib with a gap in a rectangular duct of solar air heater.
They concluded that the thermal enhancement factor was found to be highest at the attach angle of $60^{\circ}$.

Most of the previously measured data, relating to heat transfer enhancement and thermal performance in square duct and channel, were obtained based on V-rib, Vbroken rib which placed only on the walls with various parameters. The V-discrete or V-broken baffle (rib with zero thickness) placed on an adiabatic plate that inserted in the middle of the circular tube has rarely been reported, especially, numerically investigated.

Therefore, the objectives of the present work are as follows:

- Numerical study the effect of $45^{\circ} \mathrm{V}$-discrete baffle on heat transfer, pressure loss, thermal enhancement factor and flow structure in an isothermal wall circular tube

- The effect of Baffle height to Diameter Ratio (blockage ratio, b/D, BR) with single Pitch to Diameter Ratio (P/H, PR) of 1 is studied

- Flow structure and heat transfer behavior with two different flow directions; V-tip pointing downstream (V-Downstream) and V-tip pointing upstream (VUpstream) are investigated

The use of the V-discrete baffle placed periodically is expected to generate longitudinal vortex flows over the tube to better mixing of fluid flows between the core and the wall regions resulting in a higher heat transfer rate and thermal performance in the tested tube.

\section{Materials and Methods}

\section{Baffle Geometry and Arrangement}

Figure 1 shows a circular tube with a $45^{\circ} \mathrm{V}$-discrete baffle placed on a plate that inserted in the middle of the circular tube in tandem for in-line arrangement and pointing in two different directions; V-tip pointing downstream (V-Downstream) and V-tip pointing upstream (V-Upstream). The flow under consideration is expected to make a periodic flow condition in which the velocity field and thermal profile repeat itself from one cell to another. The periodically fully developed flow concepts and its solution procedure have been described in (Patankar et al., 1977). The air enters the tube at an inlet temperature, $T_{\text {in }}$ and flows over a $45^{\circ}$ in-line V-discrete baffle where $b$ is the baffle height, $D$ set to $0.05 \mathrm{~m}$, is the tube diameter and $b / \mathrm{D}$ is known as the blockage ratio, $B R$. The axial pitch, $L$ or distance between the baffle cell is set to $L=D$ in which $L / D$ is defined as the pitch spacing ratio, $P R=1$. To investigate an effect of the flow blockage ratio, $B R$ is varied in a range of $B R=0.05-0.20$ with single pitch ratio for $\alpha=45^{\circ}$ in the present investigation.

\section{Boundary Conditions}

From Promvonge et al. $(2010 ; 2012)$, inlet and outlet of the computational domain are periodic boundaries. 


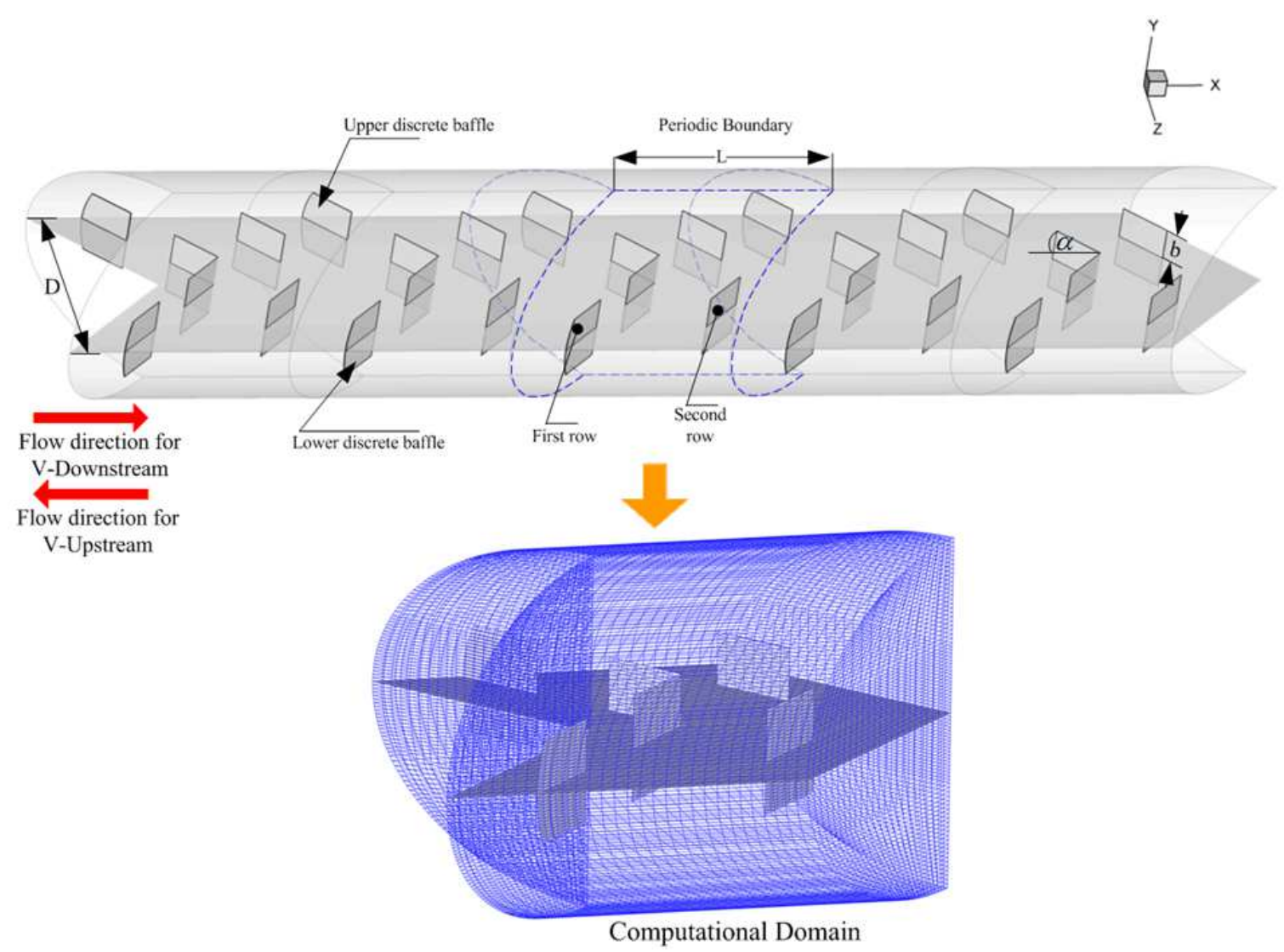

Fig. 1. Circular tube geometry and computational domain of periodic flow for V-discrete baffle

The constant mass flow rate of air with $300 \mathrm{~K}(\operatorname{Pr}=0.7)$ is assumed in the flow direction rather than constant pressure drop due to periodic flow conditions. The inlet and outlet profiles for the velocities must be identical. The physical properties of the air have been assumed to remain constant at average bulk temperature. Impermeable boundary and no-slip wall conditions have been implemented over the tube wall as well as the baffle. The constant temperature of all the tube walls is maintained at $310 \mathrm{~K}$ while the baffle and plate are assumed at adiabatic wall conditions.

\section{Mathematical Foundation}

From Promvonge et al. $(2010 ; 2012)$, the numerical model for fluid flow and heat transfer in a tube was developed under the following assumptions:

- Steady three-dimensional fluid flow and heat transfer

- The flow is laminar and incompressible

- Constant fluid properties

- Body forces and viscous dissipation are ignored

- Negligible radiation heat transfer

Based on the above assumptions, the tube flow is governed by the continuity, the Navier-Stokes equations and the energy equation. In the Cartesian tensor system these equations can be written as follows: The present problem is three-dimensional, laminar and steady. The relevant equations are the Navier-Stokes equations and energy equation. The equations in the tensor notation form are as follows.

Continuity Equation 1:

$\frac{\partial}{\partial x_{i}}\left(\rho u_{i}\right)=0$

Momentum Equation 2:

$\frac{\partial\left(\rho u_{i} u_{j}\right)}{\partial x_{j}}=-\frac{\partial p}{\partial x_{i}}+\frac{\partial}{\partial x_{j}}\left[\mu\left(\frac{\partial u_{i}}{\partial x_{j}}+\frac{\partial u_{j}}{\partial x_{i}}\right)\right]$

Energy Equation 3:

$\frac{\partial}{\partial x_{i}}\left(\rho u_{i} T\right)=\frac{\partial}{\partial x_{j}}\left(\Gamma \frac{\partial T}{\partial x_{j}}\right)$

where, $\Gamma$ is the thermal diffusivity and is given by Equation 4: 


$$
\Gamma=\frac{\mu}{\operatorname{Pr}}
$$

Apart from the energy equation discretized by the QUICK scheme, the governing equations were discretized by the second order upwind scheme, decoupling with the SIMPLE algorithm and solved using a finite volume approach (Patankar, 1980). The solutions were considered to be converged when the normalized residual values were less than $10^{5}$ for all variables but less than $10^{9}$ only for the energy equation.

Four parameters of interest in the present work are the Reynolds number, friction factor, Nusselt number and thermal enhancement factor. The Reynolds number is defined as Equation 5:

$\operatorname{Re}=\rho \bar{u} D / \mu$

The friction factor, $f$ is computed by pressure drop, $\Delta p$ across the length of the periodic tube, $L$ as Equation 6:

$f=\frac{(\Delta p / L) D}{\frac{1}{2} \rho \bar{u}^{2}}$

The heat transfer is measured by the local Nusselt number which can be written as Equation 7:

$N u_{x}=\frac{h_{x} D}{k}$

The average Nusselt number can be obtained by Equation 8:

$N u=\frac{1}{A} \int N u_{x} \partial A$

The Thermal Enhancement Factor (TEF) is defined as the ratio of the heat transfer coefficient of an augmented surface, $h$ to that of a smooth surface, $h_{0}$, at an equal pumping power and given by Equation 9:

$$
T E F=\left.\frac{h}{h_{0}}\right|_{p p}=\left.\frac{N u}{N u_{0}}\right|_{p p}=\left(N u / N u_{0}\right) /\left(f / f_{0}\right)^{1 / 3}
$$

where, $N u_{0}$ and $f_{0}$ stand for Nusselt number and friction factor for the smooth tube, respectively.

The computational domain is resolved by regular Cartesian elements. For this tube flow, however, regular grid was applied throughout the domain. A grid independence procedure was implemented by using Richardson extrapolation technique over grids with different numbers of cells. The characteristics of four grids; 53,200, 122,000, 180,400 and 321,000 cells, are used in the simulations for using the Grid Convergence
Index (GCI) (Roache, 1998). The variation in $N u$ and $f$ values for the $45^{\circ}$ in-line $\mathrm{V}$-discrete baffles at $B R=0.15$ and $R e=800$ is less than $0.25 \%$ when increasing the number of cells from 180,400 to 321,000 , thus there is no such advantage in increasing the number of cells beyond this value. Considering both convergent time and solution precision, the grid system of 180,400 cells was adopted for the current computational model.

\section{Results and Discussion}

\section{Verification of Smooth Tube}

Figure $2 a$ and $b$ show, respectively, validation of the heat transfer and friction factor of the smooth tube without baffle by comparing with the previous values under a similar operating condition. The current numerical smooth tube result is found to be in excellent agreement with exact solution values obtained from the open literature (Roache, 1998) for both the Nusselt number and the friction factor, less than $\pm 0.25 \%$ deviation. The exact solutions of the Nusselt number and the friction factor for laminar flows over smooth tube with constant wall temperature are as follows (Incropera and Dewitt, 2006) Equation 10 and 11:

$$
\begin{aligned}
& N u_{0}=3.66 \\
& f_{0}=64 / \mathrm{Re}
\end{aligned}
$$

\section{Flow Structure}

The flow configurations in a circular tube with Vdiscrete baffles placed on a plate that inserted in the middle of the tested tube can be displayed by considering the streamline plots as depicted in Fig. 3 to 7 .

Fig. 3 and 4 show the streamlines in transverse planes for V-Downstream and V-Upstream baffles, respectively, at $R e=800, B R=0.15$.

Figure $3 a$ and $4 a$ present the streamline in transverse planes while Fig. $3 b$ and $4 b$ also display the details for each plane. It is visible in Fig. 3 and 4 that there are four longitudinal main vortex flows in the tested tube. Similar two counter-rotating vortices visible on both the upper and the lower parts by reason of baffle symmetry in both cases as depicted in planes A1 to A5 and B1 to B5 for VDownstream and V-Upstream, respectively. Considering at the lower part of a pair of vortex flow, it can be concluded that the V-Upstream case produces two counter-vortex flows having a rotating direction down to the tube wall, called "common-flow-down" while the VDownstream gives opposite rotating flow direction, called "common-flow-up". The appearance of the vortex flows can help to increase higher heat transfer in the tested tube because of highly transporting the fluid from the vortex core to the wall regions. 


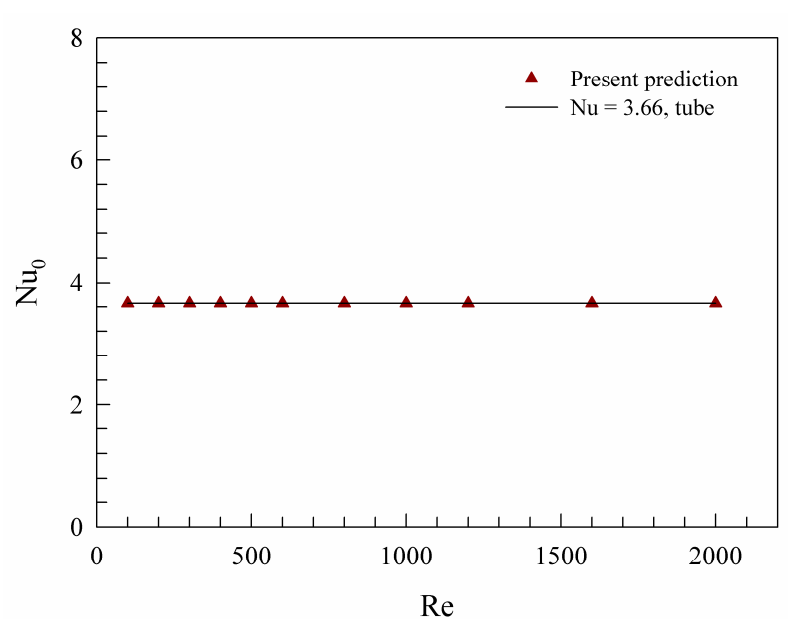

(a)

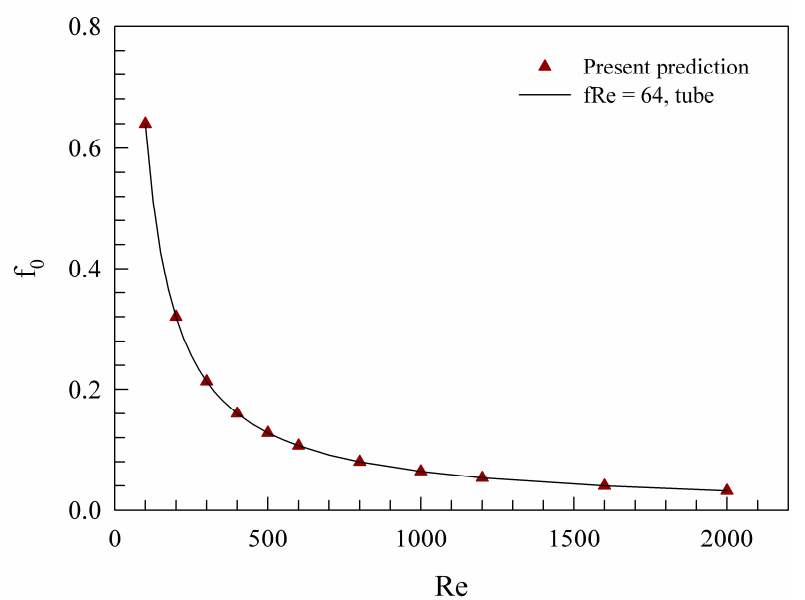

(b)

Fig. 2. Verification of (a) Nusselt number and (b) friction factor for smooth circular tube

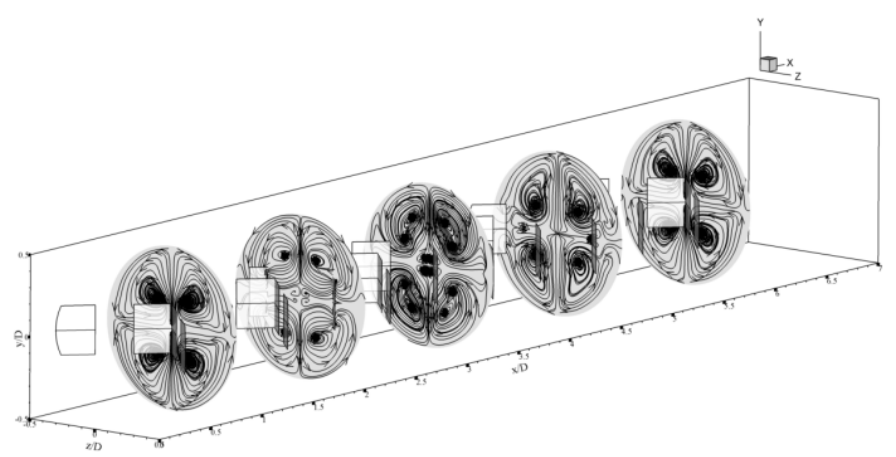

(a)
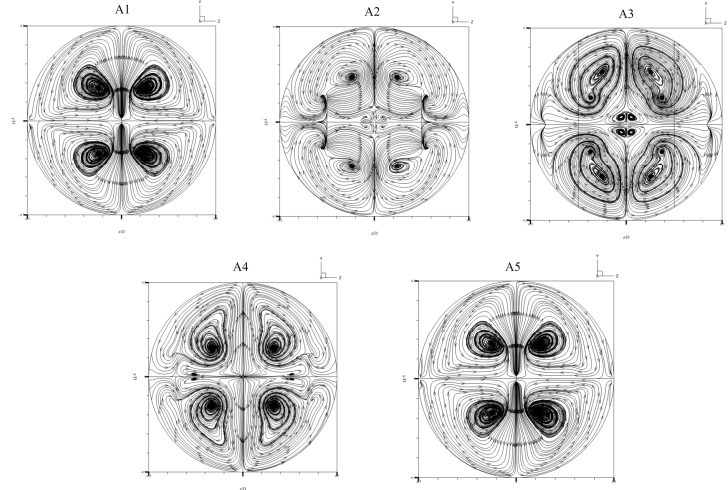

(b)

Fig. 3. (a) Streamlines in transverse planes and (b) detail of each plane for V-Downstream discrete baffles at $\mathrm{Re}=800$ and $\mathrm{BR}=0.15$

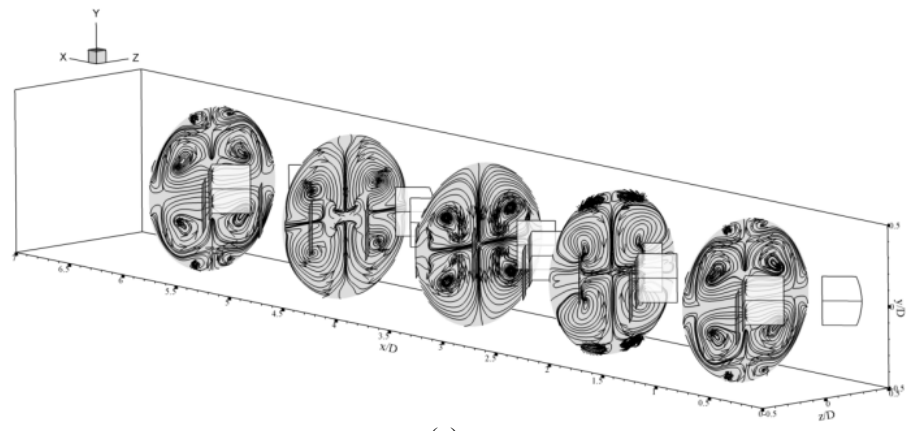

(a)
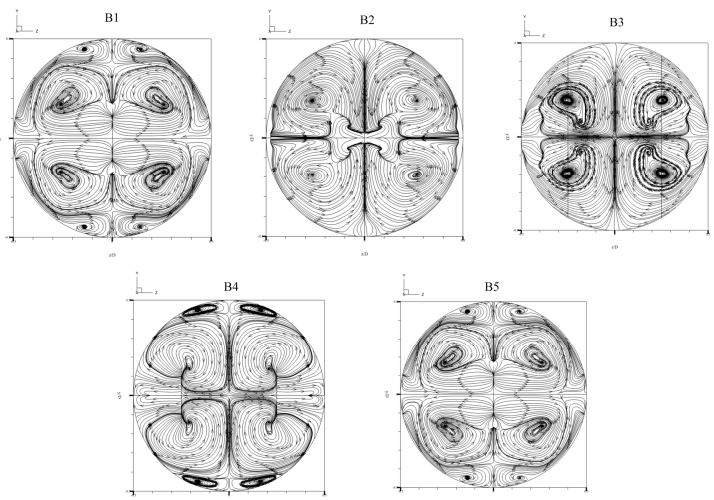

(b)

Fig. 4. (a) Streamlines in transverse planes and (b) detail of each plane for V-Upstream discrete baffles at $\mathrm{Re}=800$ and $\mathrm{BR}=0.15$

The schemes of streamlines impingement flows on the plate that installed $\mathrm{V}$-discrete baffle of the $\mathrm{V}$ Downstream and V-Upstream cases are shown in Fig. $5 a$ and $b$, respectively. There are two zones of streamlines impinging as shown in the Figures. It is visible that impinging jets happen periodically in the baffle spacing (also on the lower part due to symmetry) on both cases. 


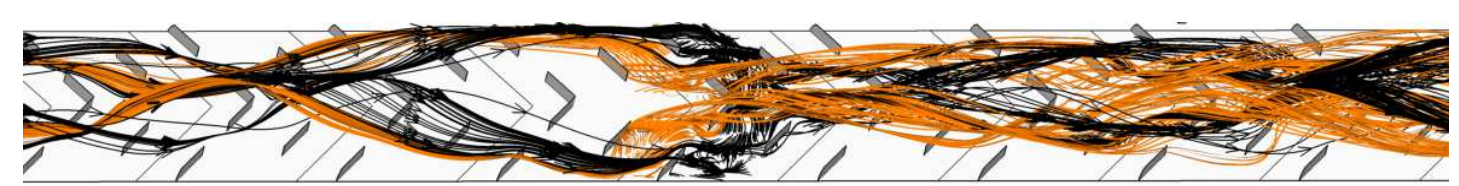

(a)

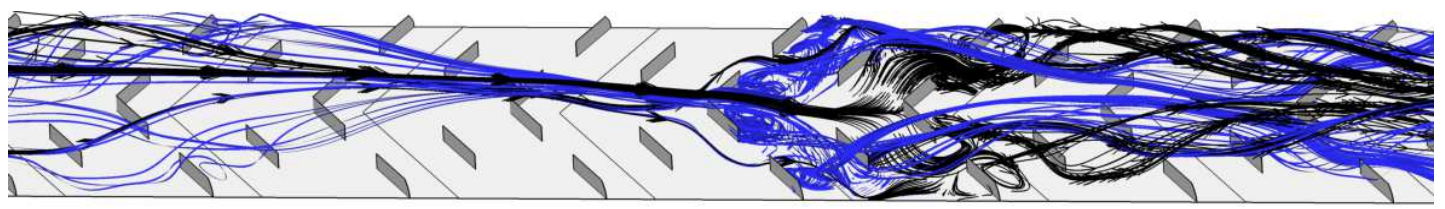

(b)

Fig. 5. Streamlines of impinging jet for (a) V-Downstream and (b) V-Upstream at $R e=800, B R=0.15$ and $P R=1$

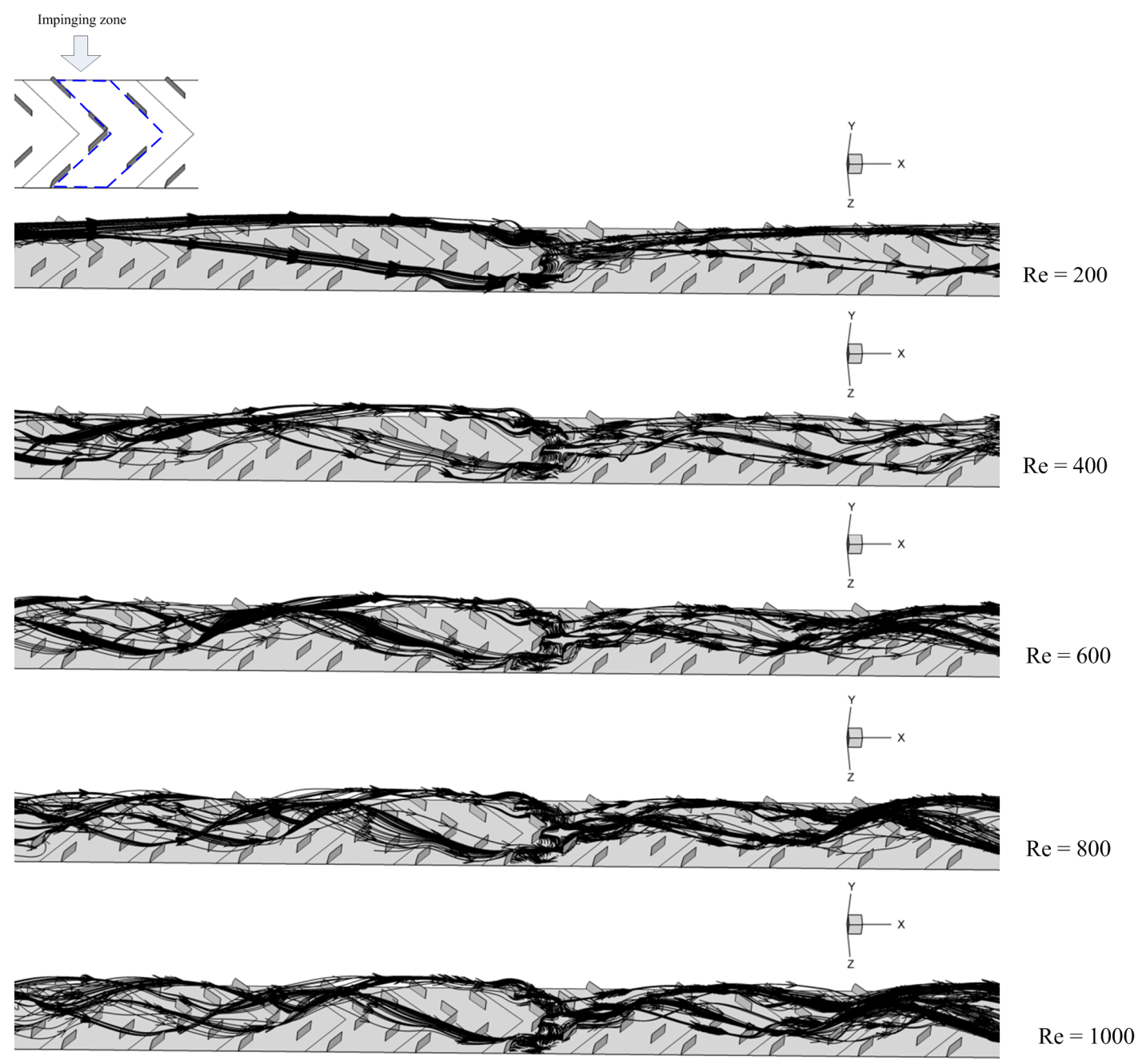

Fig. 6. Streamlines of impinging jet at various Re values for $\mathrm{V}$-Downstream discrete baffles at $\mathrm{BR}=0.15$ and $\mathrm{PR}=1$

In case of V-Downstream, before impingement, the streamline that from previous module become together and roll up to the wall of the tested tube and roll above two modules before impinging on the plate. After impingement, the jet splits over the plate and recombines into two helical streams at the nearby baffle end to create vortex flows again. The helical pitch length of the main vortex flow is about 4D before impingement and becomes shorter (about 3D) after impingement. This behavior is identical on both the upper and lower parts so two longitudinal vortices with non-uniform helical pitch are formed throughout the tube. 
In case of $\mathrm{V}$-Upstream, the impingements at the middle of zones for helical pitch length around 3D are appeared. After impingement, jets slide to sidewall position, near the plate and then roll across around 3D of V-discrete module.

The helical pitch length at different on $R e$ and $B R$ are shown in Fig. 6 and 7, respectively. Figure 6 shows the plot of streamline impinging on the plate at various $R e$ values for similar, $B R=0.15$. As seen from the figure, the higher of $R e$ result in the shorter of helical pitch length on both before and after impingement of the jets. It is found that the $R e=200$ show helical pitch length $>5 \mathrm{D}$ before impingement and 5D after impingement. Case $R e=400$, the helical pitch length presents at 4D and $3 \mathrm{D}$ before and after impingement, respectively. The helical pitch length shows the similarity pattern for cases of $R e=600$ and 800 , around $3 \mathrm{D}$ and $2 \mathrm{D}$ for before and after impingement, respectively. The $R e=1000$ gives the shortest helical pitch length around 2D before impingement and 1D after impingement.

Figure 7, the plots of streamline are presented at various $B R$ s with single $R e$ value, $R e=500$. It is visible that the higher of $B R$ lead to the decrease in helical pitch length on both before and after impingement. The $B R=$ 0.20 case shows the shortest helical pitch length around $2 \mathrm{D}$ and 1D for before and after impingement, respectively, while the $B R=0.05$ provides very long helical pitch length $>6 \mathrm{D}$ on both before and after impingement. The unpredictability of the helical pitch length depend on $R e, B R$ and flow direction of the Vdiscrete baffle.

\section{Heat Transfer Behavior}

Local $N u_{\mathrm{x}}$ contours for the tube wall with the $\mathrm{V}$ discrete baffles at $R e=800$ and $B R=0.15$ are presented in Fig. $8 a$ and $b$ for V-Downstream and V-Upstream, respectively. In these figures, it is found that the $\mathrm{V}$ Downstream case shows higher $N u_{\mathrm{x}}$ values over the smooth tube with no baffle, especially, on the side that does not close to the installed plate while the VUpstream case provides opposite result about enhancement of heat transfer area.

The variation of the average $N u / N u_{0}$ ratio with Reynolds number at different $B R \mathrm{~s}$ is depicted in Fig. 9. It appears that the $N u / N u_{0}$ value tends to increase with the rise of Reynolds number for all cases. The higher on $B R$ value results in the augment in the $N u / N u_{0}$ value. At the lowest $B R, B R=0.05$, the $N u / N u_{0}$ of V-Upstream and VDownstream provides nearly values while a case of $B R=$ $0.10, \mathrm{~V}$-Upstream give higher heat transfer rate than the V-Downstream at $R e>300$. Cases of $B R=0.15$ and 0.20, the $N u / N u_{0}$ of $\mathrm{V}$-Downstream show higher than $\mathrm{V}$ Upstream for all Re values.

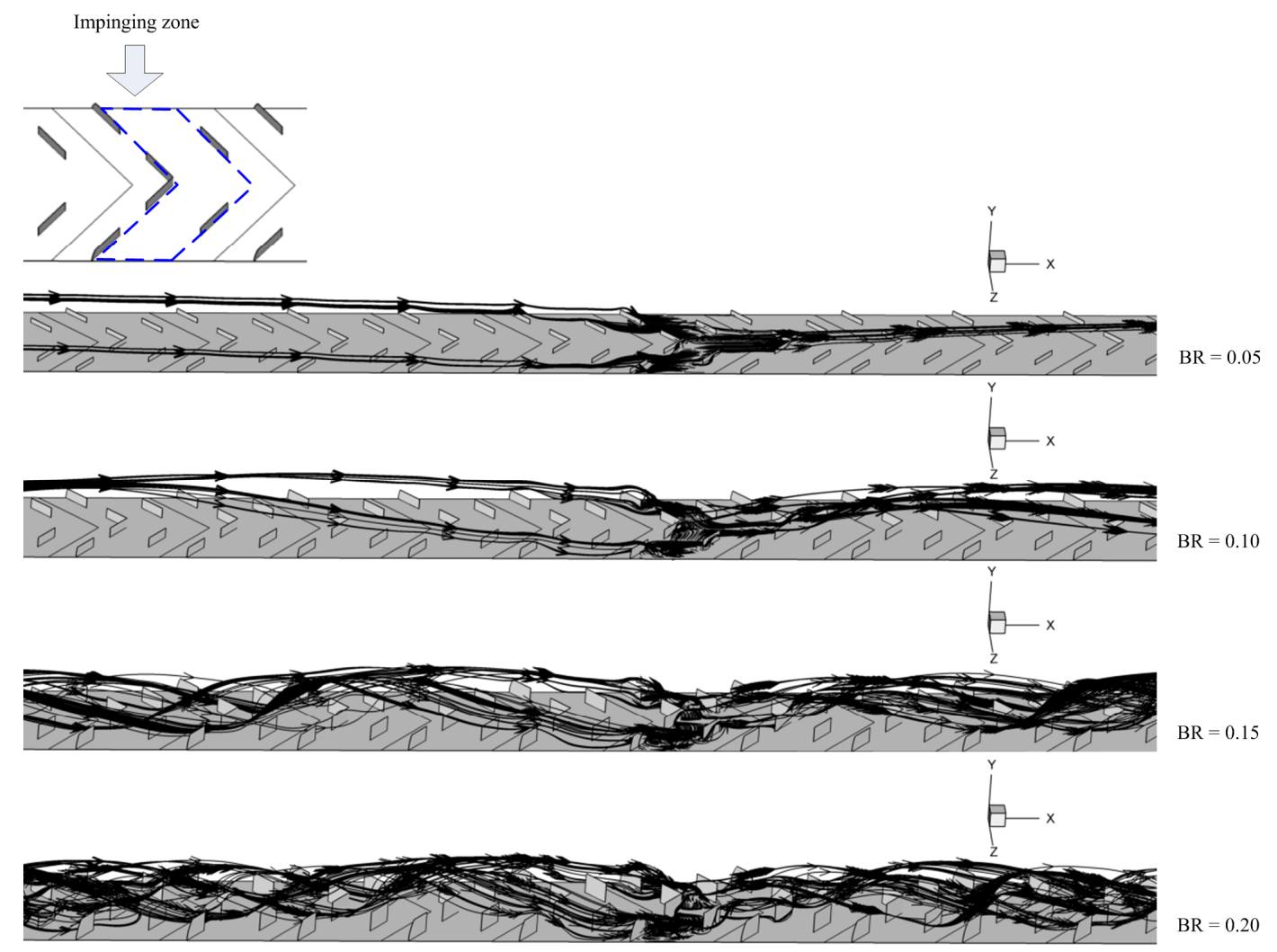

Fig. 7. Streamlines of impinging jet at various $B R$ values for V-Downstream discrete baffles at $R e=500$ and $P R=1$ 


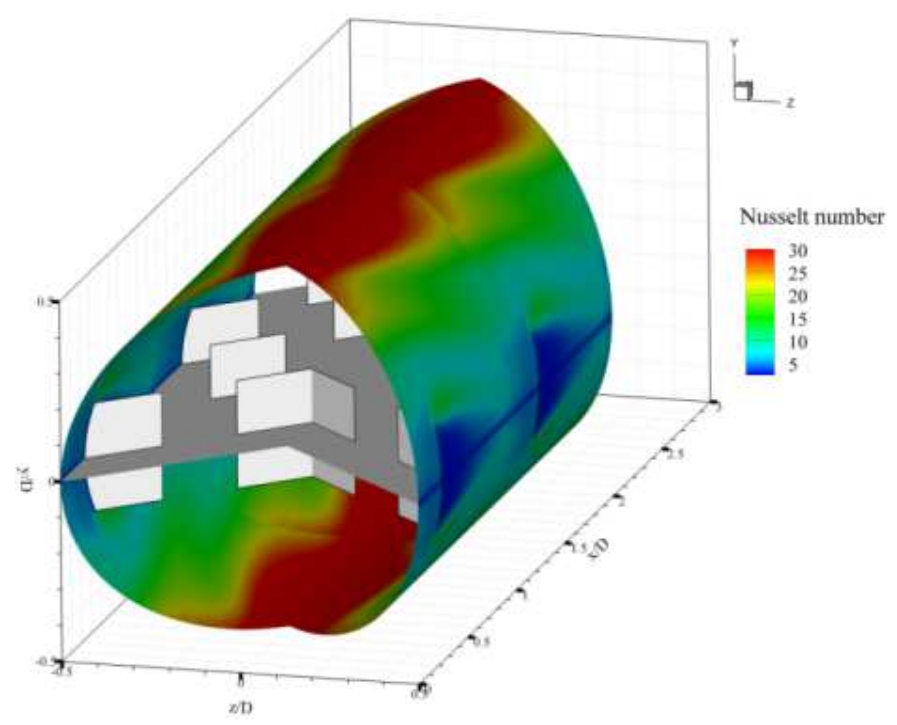

(a)

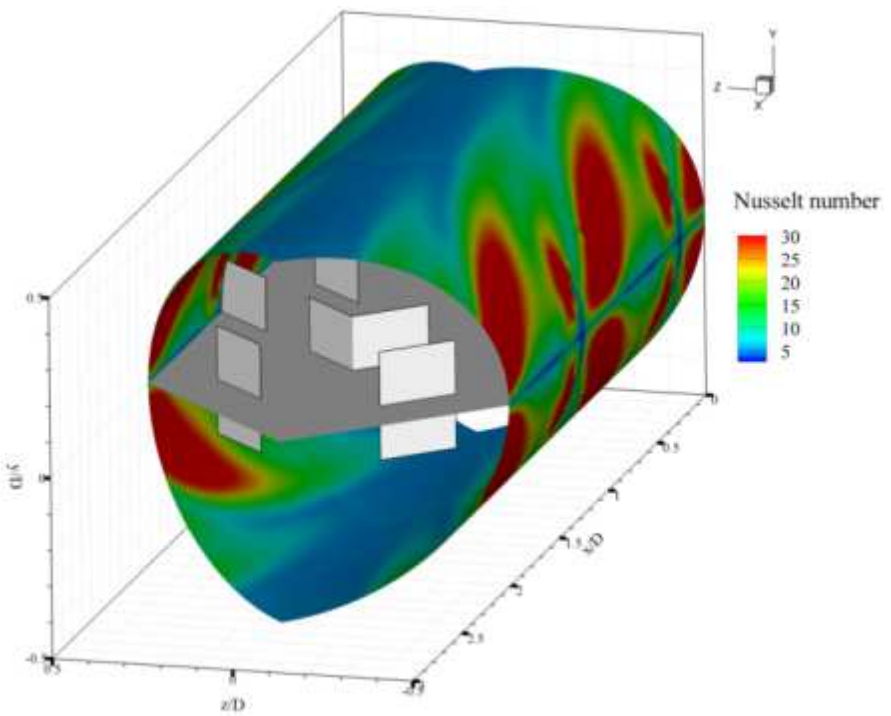

(b)

Fig. 8. $\mathrm{Nu}_{\mathrm{x}}$ Contours for (a) V-Downstream and (b) $\mathrm{V}$-Upstream at $R e=800$ and $B R=0.15$

Thus, the generation of longitudinal vortex flows from using the V-discrete baffles as well as the role of better fluid mixing and the impingement is the main cause for the augmentation in heat transfer of the tested tube. The use of the V-discrete baffle with range studied gives heat transfer rate of about 1.2-8.3 times higher than the plain circular tube with no baffle.

\section{Pressure Loss}

Figure 10 displays the variation of the friction factor, $f l f_{0}$ with Reynolds number values for various $B R \mathrm{~s}$ of $\mathrm{V}$-discrete baffle. In the figure, it is visible that the $f l f_{0}$ tends to increase with the rise of $R e$ and $B R$ values. Cases of $B R=0.05$ and 0.10 , the V-Upstream give a higher $\mathrm{f} / f_{0}$ that V-Downstream for all Reynolds number while $B R=0.15$ and 0.20 cases, the V-Downstream show higher values of friction factor ratios than the V-Upstream at $R e>300$. The use of the Vdiscrete baffle leads to a considerable increase in friction factor in comparison with the plain tube with no baffle. The $f f f_{0}$ values for all cases are found to be about 3-36 times over the smooth tube depending on the $B R$, flow direction and Reynolds number values.

\section{Thermal Enhancement Factor}

Figure 11 exhibits the variation of thermal Enhancement Factor $(T E F)$ for air flowing in the baffled circular tube. The enhancement factor of both V-discrete baffles tends to increase with the rise of $R e$ and $B R$ values. All of the Vdiscrete baffles provide the highest enhancement factor at the highest $R e, R e=1200$. 


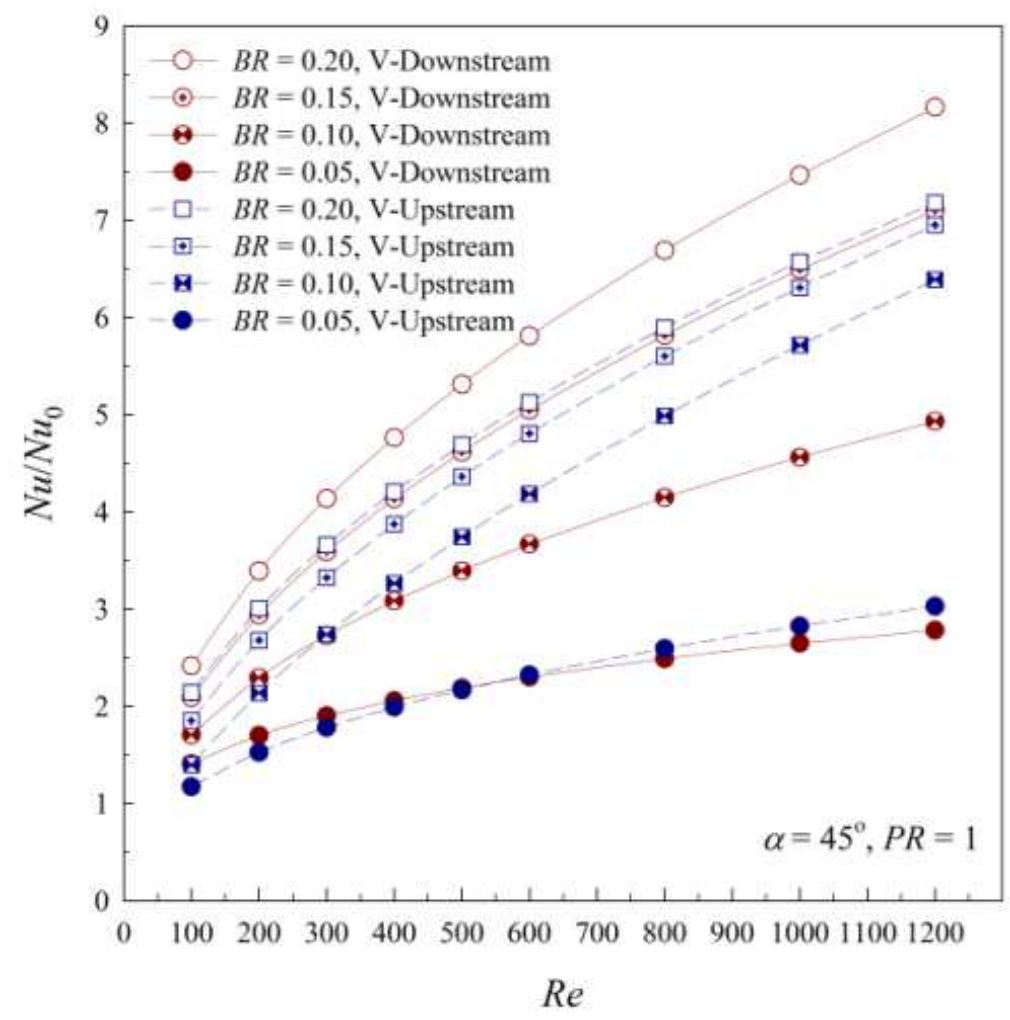

Fig. 9. Variation of $N u / N u_{0}$ with Reynolds number for various baffles $B R \mathrm{~s}$

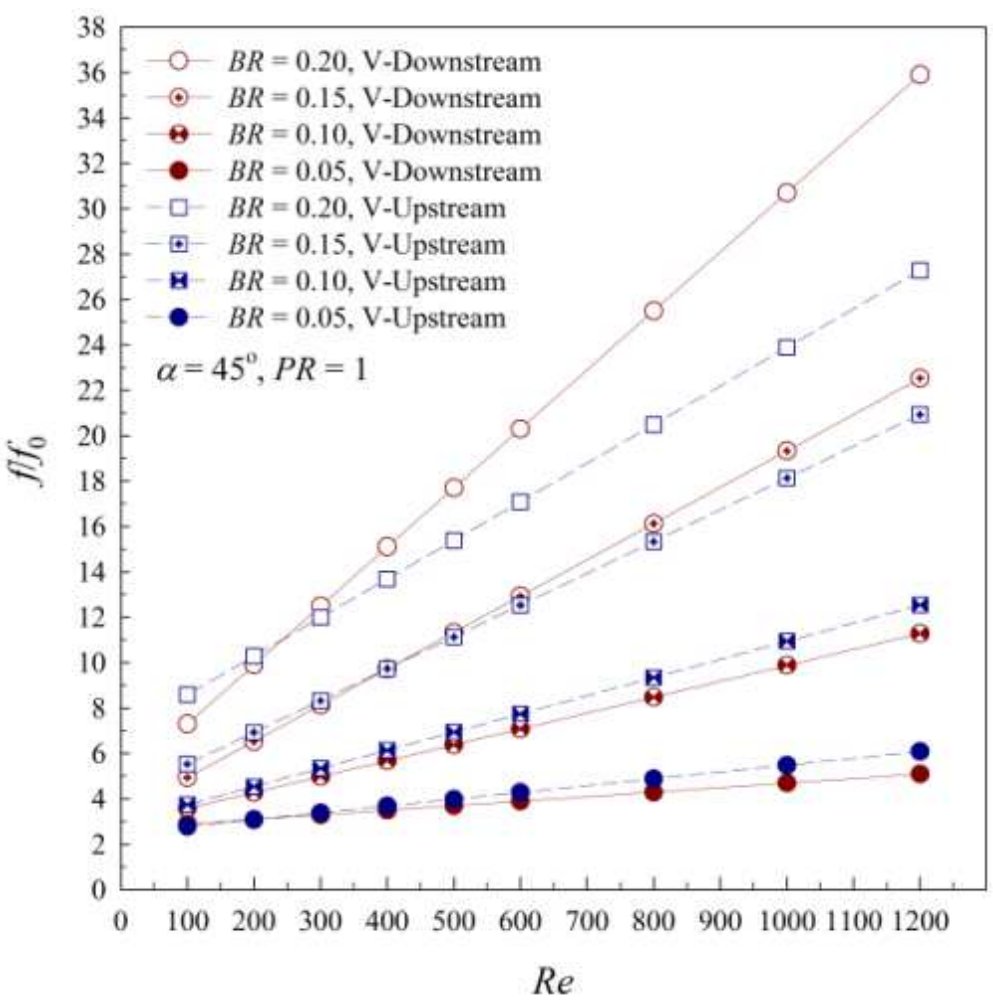

Fig. 10. Variation of $f \mid f_{0}$ with Reynolds number for baffles at various $B R$ s 


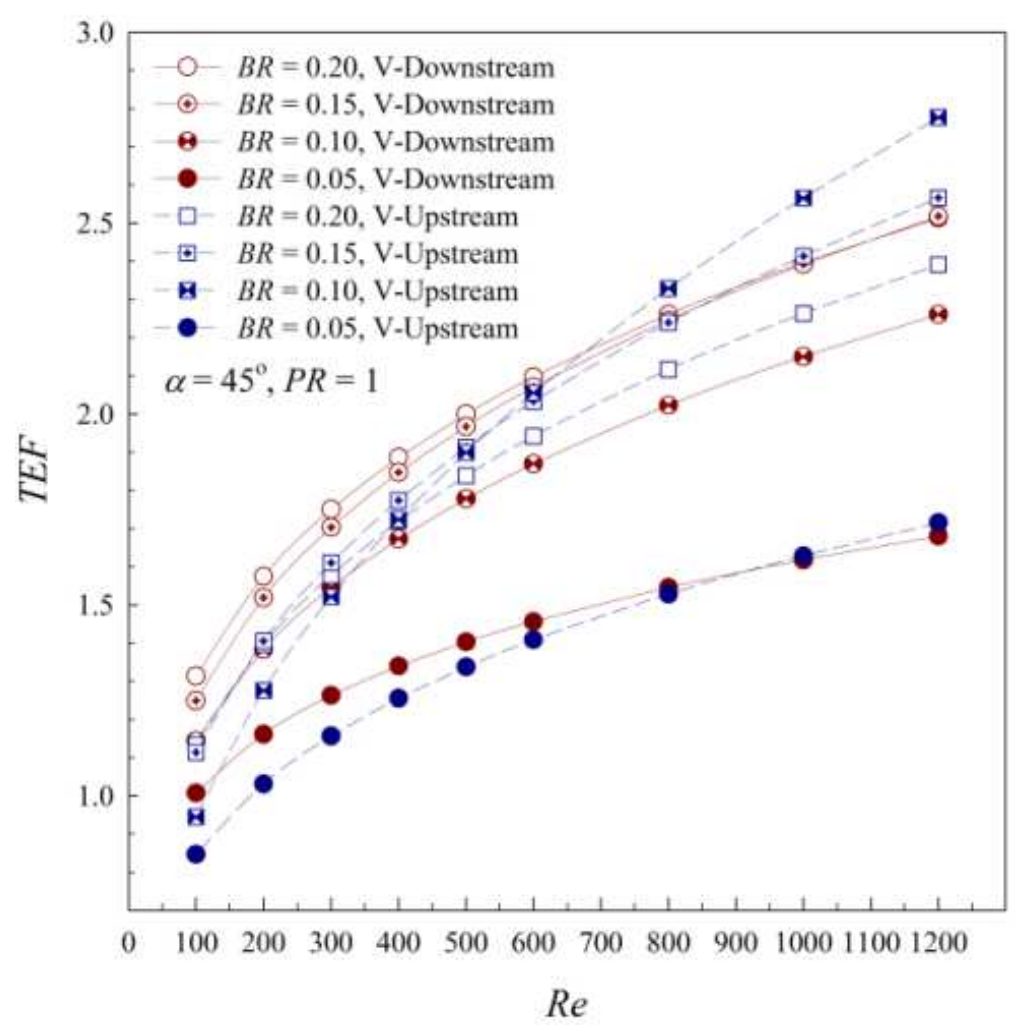

Fig. 11. Comparison of thermal enhancement factor at various $B R \mathrm{~s}$

The enhancement factors of both the V-discrete baffles are seen to be above unity for cases and vary between 1.0 and 2.75 , depending on the $B R$, flow direction and $R e$ values.

\section{Conclusion}

Fully developed periodic laminar flow configurations and heat transfer characteristics in a circular tube fitted with $45^{\circ} \mathrm{V}$-Downstream and V-Upstream discrete baffle elements in tandem, in-line arrangements placed on a plate that inserted in the middle of the tested tube have been investigated numerically:

- The longitudinal vortex flows created by using the $45^{\circ}$ V-discrete baffle cases help to induce impingement flows on the wall in the inter baffle cavity leading to a drastic increase in heat transfer in the circular tube on both V-Downstream and VUpstream cases

- The augmentation on heat transfer is about 1.2-8.3 time over than smooth circular tube for using the VDownstream and V-Upstream discrete baffle cases in a range studied, $B R=0.05-0.20$ with single pitch ratio, $P R=1$

- The pressure loss in the range studied is ranging from 3 to 36 times above the smooth plain tube
- Thermal enhancement factors for both the Vdiscrete baffles are found to be in a range of 1.002.75 and the maximum $T E F$ found at $B R=0.10$ for V-Upstream case at the highest $R e$

- It is found that the V-Upstream produces two counter-vortex flows having a rotating direction down to the wall, called "common-flow-down" while the V-downstream generates opposite rotating flow direction, called "common-flow-up"

- The flow directions; V-Downstream and VUpstream, create the different of impingement area (higher heat transfer area) on the tube wall, near the plate that installed V-discrete baffle for VDownstream case and far from the plate in case of V-Upstream

- The V-discrete baffle givesa lower pressure drop than solid V-baffle and also comfortable for installed than the baffle placed on tube wall (Jedsadaratanachai and Promvonge, 2012)

\section{Acknowledgement}

The funding of this study is supported by King Mongkut's Institute of Technology Ladkrabang, Thailand. The authors would like to thank Assoc. Prof. Dr. Pongjet Promvonge for suggestions. 


\section{Author's Contributions}

All authors equally contributed in this work.

\section{Ethics}

This article is original and contains unpublished material. The corresponding author confirms that all of the other authors have read and approved the manuscript and no ethical issues involved.

\section{References}

Chang, S.W., T.L. Yang, T.M. Liou and G.F. Hong, 2009. Heat transfer of rotating rectangular duct with compound scaled roughness and V-ribs at high rotation numbers. Int. J. Thermal Sci., 48: 174-187. DOI: $10.1016 /$ j.ijthermalsci.2008.03.001

Chang, S.W., T.M. Liou, K.F. Chiang and G.F. Hong, 2008. Heat transfer and pressure drop in rectangular channel with compound roughness of $\mathrm{V}$-shaped ribs and deepened scales. Int. J. Heat Mass Transfer, 51: 457-468.

DOI: 10.1016/j.ijheatmasstransfer.2007.05.010

Fu, W.L., L.M. Wright and J.C. Han, 2006. Heat transfer in two-pass rotating rectangular channels $(\mathrm{AR}=2: 1)$ with discrete ribs. J. Thermophysics Heat Transfer, 20: 569-582. DOI: $10.2514 / 1.19468$

Giovanni, T., 2004. Heat transfer in rectangular channels with transverse and $\mathrm{V}$-shaped broken ribs. Int. J. Heat Mass Transfer, 47: 229-243. DOI: $10.1016 / \mathrm{S} 0017-9310(03) 00414-9$

Han, J.C. and Y.M. Zhang, 1992. High performance heat transfer ducts with parallel broken and $\mathrm{V}$-shaped broken ribs. Int. J. Heat Mass Transfer, 35: 513-523. DOI: 10.1016/0017-9310(92)90286-2

Incropera, F.P. and P.D. Dewitt, 2006. Introduction to Heat Transfer. 5th Edn., John Wiley and Sons Inc, ISBN-10: 0471457272, pp: 912.

Jedsadaratanachai, W. and P. Promvonge, 2012. Laminar flow behavior and heat transfer in circular tube with various winglet turbulators. Proceedings of the 27th Conference of the Mechanical Engineering Network of Thailand, (ENT'12).

Karwa, R. and G. Chitoshiya, 2013. Performance study of solar air heater having v-down discrete ribs on absorber plate. Energy, 55: 939-955. DOI: $10.1016 /$ j.energy.2013.03.068

Lee, E., L.M. Wright and J.C. Han, 2005. Heat transfer in rotating rectangular channels with $\mathrm{V}$-shaped and angled ribs, J. Thermophysics Heat Transfer, 19: 48-56. DOI: $10.2514 / 1.9065$
Momin, A.M.E., J.S. Saini and S.C. Solanki, 2002. Heat transfer and friction in solar air heater duct with Vshaped rib roughness on absorber plate. Int. J. Heat Mass Transfer, 45: 3383-3396.

DOI: $10.1016 / \mathrm{S} 0017-9310(02) 00046-7$

Muluwork, K.B., 2000. Investigations on fluid flow and heat transfer in roughened absorber solar heaters. Ph.D. Theses, IIT, Roorkee.

Patankar, S.V., 1980. Numerical Heat Transfer and Fluid Flow. 1st Edn., Hemisphere Publishing Corporation, Washington, ISBN-10: 0070487405, pp: 197.

Patankar, S.V., C.H. Liu and E.M. Sparrow, 1977. Fully developed flow and heat transfer in ducts having streamwise-periodic variations of cross-sectional area. J. Heat Transfer, 99: 180-186.

DOI: $10.1115 / 1.3450666$

Peng, W., P.X. Jiang, Y. P. Wang and B.Y. Wei, 2011. Experimental and numerical investigation of convection heat transfer in channels with different types of ribs. Applied Thermal Eng., 31: 2702-2708. DOI: 10.1016/j.applthermaleng.2011.04.040

Promvonge, P., W. Jedsadaratanachai and S. Kwankaomeng, 2010. Numerical study of laminar flow and heat transfer in square channel with $30^{\circ}$ inline angled baffle turbulators. Applied Thermal Eng., 30: 1292-1303.

DOI: 10.1016/j.applthermaleng.2010.02.014

Promvonge, P., W. Jedsadaratanachai, S. Kwankaomeng and C. Thianpong, 2012. 3D simulation of laminar flow and heat transfer in V-baffled square channel. Int. Commun. Heat Mass Transfer. 39: 85-93. DOI: 10.1016/j.icheatmasstransfer.2011.09.004

Roache, P.J., 1998. Verification and Validation in Computational Science and Engineering. Hermosa, Albuquerque, NM, ISBN-10: 0913478083, pp: 446.

Singh, S., S. Chander and J.S. Saini, 2012. Investigations on thermo-hydraulic performance due to flowattack-angle in $\mathrm{V}$-down rib with gap in a rectangular duct of solar air heater. Applied Energy, 97: 907-912. DOI: 10.1016/j.apenergy.2011.11.090

Taslim, M.E., D.M. Kercher and T. Li, 1996. Experimental heat transfer and friction in channels roughened with angled, V-shaped and discrete ribs on two opposite walls. J. Turbomach, 118: 20-28. DOI: $10.1115 / 1.2836602$ 